\title{
The fragranced products phenomenon: air quality and health, science and policy
}

\author{
Anne Steinemann ${ }^{1,2}$ \\ Received: 26 August 2020 / Accepted: 31 August 2020 / Published online: 19 September 2020 \\ (C) The Author(s) 2020
}

\begin{abstract}
Fragrance is used in consumer products around the world. However, fragrance has been associated with adverse effects on indoor and outdoor air quality and human health. Questions arise, such as the following: Why does fragrance in products pose problems? What are sources of emissions and exposures? What are health and societal effects? What are possible solutions? This paper examines the issue of fragranced consumer products and its science and policy dimensions, with a focus on the implications for air quality and human health. Results include new findings and new questions for future research directions.
\end{abstract}

Keywords Fragranced consumer products · Volatile organic compounds · Fragrance-free policies · Hazardous air pollutants · Indoor air quality $\cdot$ Outdoor air quality

\section{Introduction}

Fragrance has been used for thousands of years, with the intent to create a more aesthetically pleasing environment and more popular consumer products. However, in recent years, fragrance in products has been associated with adverse effects on air quality and health, despite extensive tests for safety (IFRA 2020a). What is perplexing is why products designed to be positive may be creating unintended effects.

As background, most of our exposure to pollutants occurs indoors. A primary source of indoor air pollutants is fragranced consumer products, such as air fresheners and cleaning supplies. Further, fragranced products have been implicated as a contributor to outdoor pollutants. However, indoor environments and fragranced product emissions are essentially unregulated. Also, fragranced product ingredients are not required to be fully and specifically disclosed, not on labels, safety data sheets, or elsewhere. Therefore, an important source of air pollutants and exposures is largely unregulated, and the emissions and ingredients are largely unknown.

Anne Steinemann

anne.steinemann@unimelb.edu.au

1 Department of Infrastructure Engineering, Melbourne School of Engineering, The University of Melbourne, Parkville, Victoria 3010, Australia

2 College of Science and Engineering, James Cook University, Townsville, Queensland 4814, Australia
Yet exposures have been associated with a range of health problems, such as breathing difficulties and headaches, in nearly a third of the general population in four countries representing three continents.

This paper analyzes and synthesizes data and research findings on the fragranced products phenomenon. A goal is to provide an integrated understanding of the scientific foundations and policy implications for air quality and health. To do so, it investigates the following questions: How prevalent is product use and exposure? What health problems are associated with exposure? What volatile ingredients are emitted from the products? Are ingredients disclosed? Are green, organic, and natural products any different? What strategies can reduce emissions and exposures? Results point to new insights and new directions for research and, ultimately, new knowledge for understanding and reducing potential effects of fragranced consumer products on air quality and health.

To begin, this paper provides some definitions:

A "fragrance" is a scent and, despite its singular name, it is a formulation of dozens of chemicals, such as volatile organic compounds (VOCs). Nearly 4000 ingredients have been documented for use in the composition of a fragrance (IFRA 2020 b). A fragrance is generally intended to "provide an aroma, to mask an odor, or both" (Steinemann 2019a).

A "fragranced consumer product" (or "fragranced product" for brevity) is a product that "contains an added fragrance or that is largely comprised of fragrance" (Steinemann 2019a). Fragranced products cover hundreds of everyday items, such as air fresheners, deodorizers, cleaning supplies, laundry 
detergents, fabric softeners, essential oils, candles, soaps, personal care products, colognes, and hand sanitizers.

A distinction is made herein between a cosmetic item termed fragrance (such as a perfume or cologne) versus a chemical mixture termed fragrance that is added to a product. This article focuses on the latter, although a fragrance (perfume, cologne) is one of the hundreds of types of fragranced consumer products.

"Fragrance sensitivity" is a health condition characterized by one or more types of adverse health effects from exposure to one or more types of fragranced consumer products (Steinemann 2019b).

\section{International studies of fragranced consumer products: emissions, exposures, and effects}

Nationally representative population-based studies were conducted across four countries-the United States (US), Australia (AU), the United Kingdom (UK), and Sweden (SE) - to investigate fragranced product emissions, exposures, and effects. The studies used cross-sectional surveys of adults ages 18-65, with a questionnaire in the native language as the survey instrument. Sample populations $(n=$ $1137,1098,1100,1100$, respectively) were representative of the general populations according to age, gender, and region (confidence level $=95 \%$, margin of error $=3 \%$ for all studies) . Using randomized participant recruitment, the surveys drew upon large web-based panels (with over 5,000,000; 200,000; 900,$000 ; 60,000$ participants, respectively). In addition to the general population, the survey also investigated effects on vulnerable sub-populations, such as asthmatic individuals and autistic individuals. The surveys were performed in June 2016 (US, AU, UK) and June 2017 (SE). The survey response rates were $94 \%, 93 \%, 97 \%$, and $92 \%$ (respectively), and all responses were anonymous. (For additional survey details, see Steinemann 2016, 2017a, 2018c, d, 2019b.)

The studies investigated the following types of fragrance products, exposures, and effects.

Fragranced products were categorized as follows: “(a) air fresheners and deodorizers (e.g., sprays, solids, oils, disks); (b) personal care products (e.g., soaps, hand sanitizer, lotions, deodorant, sunscreen, shampoos); (c) cleaning supplies (e.g., all-purpose cleaners, disinfectants, dishwashing soap); (d) laundry products (e.g., detergents, fabric softeners, dryer sheets); (e) household products (e.g., scented candles, restroom paper, trash bags, baby products); (f) fragrance (e.g., perfume, cologne, after-shave, essential oils); and (g) other."

Exposure contexts included the following: "air fresheners or deodorizers used within indoor environments; scented laundry products coming from a dryer vent; being in a room after it was cleaned with scented cleaning products; being near someone wearing a fragranced product; and exposure to other types of fragranced consumer products."

Health effects were categorized as follows: "(a) migraine headaches; (b) asthma attacks; (c) neurological problems (e.g., dizziness, seizures, head pain, fainting, loss of coordination); (d) respiratory problems (e.g., difficulty breathing, coughing, shortness of breath); (e) skin problems (e.g., rashes, hives, red skin, tingling skin, dermatitis); (f) cognitive problems (e.g., difficulties thinking, concentrating, or remembering); (g) mucosal symptoms (e.g., watery or red eyes, nasal congestion, sneezing); (h) immune system problems (e.g., swollen lymph glands, fever, fatigue); (i) gastrointestinal problems (e.g., nausea, bloating, cramping, diarrhea); (j) cardiovascular problems (e.g., fast or irregular heartbeat, jitteriness, chest discomfort); (k) musculoskeletal problems (e.g., muscle or joint pain, cramps, weakness); and (l) other."

For fragrance sensitivity, the survey asked, "Do you experience any health problems when exposed to (specific fragranced product or exposure context)?" If the respondent answered yes, the survey then asked the respondent to specify which health problem(s) they experienced. An individual was considered to characterize fragrance sensitivity if they reported one or more types of health problems from exposure to one or more types of fragranced consumer products or exposure contexts.

The study also identified vulnerable populations, such as asthmatic individuals and autistic individuals.

For asthmatic individuals, the survey asked, "Has a doctor or health care professional ever told you that you have asthma or an asthma-like condition?" If the respondent answered yes, the survey then asked to specify which one or both. For this study, "asthmatic individuals" are considered to be medically diagnosed with asthma, an asthma-like condition, or both.

For autistic individuals, the survey asked, "Has a doctor or health care professional ever told you that you have autism or autism spectrum disorder?" If the respondent answered yes, the survey then asked to specify which one or both. For this study, "autistic individuals" are considered to be medically diagnosed with autism, an autism spectrum disorder, or both.

Results are provided below, with averages across the countries provided as single percentage, and with individual country results (US, AU, UK, SE) provided as a series of four percentages, respectively. Complete results with data and statistics, for each country, each sup-population, and each question, are provided in Steinemann (2016, 2017a, 2018a, b, c, d, 2019a, b, c).

\section{Pervasiveness of product use and exposure}

Fragranced consumer products are used around the world. Exposure is common, both direct and indirect, voluntary and involuntary. 
Across the four countries, $99.1 \%$ of the population is exposed to fragranced products, at least once a week, from their own use (98.3\%), from others' use (90.6\%), or from either or both $(99.1 \%)$. For individual countries: from own use $(98.3 \%$, $98.0 \%, 98.5 \%, 98.5 \%)$, from others' use $(92.1 \%, 88.1 \%$, $89.0 \%, 93.3 \%)$, or from either or both $(99.0 \%, 98.5 \%$, $99.3 \%, 99.5 \%)$.

Specific product results, for population exposure at least once a week, are as follows: air fresheners and deodorizers, $74.7 \%$; personal care products, $93.2 \%$; cleaning supplies, $86.2 \%$; laundry products, $87.1 \%$; household products, $79.1 \%$; fragrance, $82.8 \%$; and other products, $3.7 \%$.

The problem of widespread exposure $(90.6 \%)$ to others' use of products gives rise to the problem of "secondhand scents": indirect or involuntary exposure to fragranced products (Steinemann 2019a). Consequences of secondhand scents include, for example, restricted access in society, health risks, lost workdays and lost jobs, and negative effects in daily life and living situations.

\section{Prevalence of fragrance sensitivity}

Among the general population, across the four countries, $32.2 \%$ of adults on average $(34.7 \%, 33.0 \%, 27.8 \%, 33.1 \%)$ report fragrance sensitivity; that is, adverse health effects from exposure to fragranced consumer products.

Among vulnerable sub-populations, the prevalence of fragrance sensitivity is higher. For instance, $57.8 \%$ of asthmatic individuals and $75.8 \%$ of autistic individuals report adverse effects from fragranced products.

\section{Fragranced product exposures}

Fragranced product exposures, with associated frequencies of reports of health problems, include but are not limited to the following:

General population: air fresheners and deodorizers, $17.4 \%$ $(20.4 \%, 16.4 \%, 15.5 \%, 17.3 \%)$; fragranced laundry products coming from a dryer vent, $7.6 \%(12.5 \%, 6.1 \%, 6.0 \%, 5.6 \%)$; being in a room after it has been cleaned with fragranced products, $15.7 \%(19.7 \%, 15.3 \%, 14.0 \%, 13.8 \%)$; being near someone who is wearing a fragranced product, $20.1 \%(23.6 \%$, $19.4 \%, 13.7 \%, 23.5 \%$ ); and other types of fragranced consumer products, $18.6 \%(22.3 \%, 20.3 \%, 13.9 \%, 17.9 \%)$.

For vulnerable sub-populations, the exposures and associated frequencies of reports of health problems (\% relative to each sub-population) include the following:

Asthmatic individuals: air fresheners and deodorizers (36.7\%), fragranced laundry products coming from a dryer vent $(18.1 \%)$, being in a room after it has been cleaned with fragranced products $(32.9 \%)$, being near someone who is wearing a fragranced product (38.7\%), and other types of fragranced consumer products $(37.5 \%)$.

Autistic individuals: air fresheners and deodorizers $(62.9 \%)$, fragranced laundry products coming from a dryer vent $(57.5 \%)$, being in a room after it has been cleaned with fragranced products $(65.9 \%)$, being near someone who is wearing a fragranced product $(60.5 \%)$, and other types of fragranced consumer products $(64.3 \%)$.

Vulnerable sub-populations have a higher frequency of reports of health problems than the general population, across all exposure contexts. For instance, when exposed to air fresheners or deodorizers, $36.7 \%$ of asthmatic individuals and $62.9 \%$ autistic individuals report health problems, compared with $17.4 \%$ of the general population.

Each of these exposure contexts represents a potentially involuntary exposure, and one that can pose risks to an individual's health and their ability to have access and opportunities in society. Further, all types of fragranced products and exposure contexts studied were associated with adverse health effects.

\section{Health problems}

Across the general population in the four countries, the frequency and types of adverse health effects associated with fragranced product exposures include the following: respiratory problems, $16.7 \%(18.6 \%, 16.7 \%, 11.6 \%, 20.0 \%)$; mucosal symptoms, $13.2 \%(16.2 \%, 14.0 \%, 9.2 \%, 13.5 \%)$; migraine headaches, $12.6 \%$ (15.7\% $10.0 \% \quad 8.4 \% 16.1 \%)$; skin problems, $9.1 \%$ (10.6\% 9.5\% 9.8\% 6.5\%); asthma attacks, $7.0 \%$ (8.0\% 7.6\% 6.8\% 5.5\%); neurological problems, $5.1 \%$ (7.2\% $4.5 \% 3.7 \%$ 5.0\%); cognitive problems, $4.3 \%$ (5.8\% $4.1 \%$ $2.8 \% 4.5 \%)$; gastrointestinal problems, $3.8 \%(5.5 \% 3.3 \%$ $3.0 \% 3.5 \%)$; cardiovascular problems, $3.2 \%(4.4 \% 3.0 \%$ $3.2 \% 2.1 \%)$; immune system problems, $2.7 \%$ (4.0\% $3.3 \%$ $1.9 \% 1.5 \%)$; musculoskeletal problems, $2.5 \%$ (3.8\% 2.6\% $2.0 \% 1.5 \%)$; and other, $2.0 \%$ (1.7\% 1.9\% 2.1\% 2.2\%).

For vulnerable sub-populations, the frequencies of the types of adverse health effects (\% relative to that sub-population) include the following:

For asthmatic individuals: respiratory problems $(37.7 \%)$, mucosal symptoms $(25.4 \%)$, asthma attacks $(25.0 \%)$, migraine headaches $(22.6 \%)$, skin problems $(17.1 \%)$, neurological problems $(10.2 \%)$, cognitive problems $(9.8 \%)$, gastrointestinal problems $(8.6 \%)$, cardiovascular problems $(7.9 \%)$, immune system problems $(6.5 \%)$, musculoskeletal problems $(6.5 \%)$, other $(1.6 \%)$.

For autistic individuals: respiratory problems $(44.7 \%)$, migraine headaches $(42.9 \%)$, mucosal symptoms $(42.1 \%)$, skin problems $(39.7 \%)$, asthma attacks $(35.9 \%)$, cardiovascular problems $(34.3 \%)$, neurological problems (34.3\%), musculoskeletal problems $(34.1 \%)$, cognitive problems $(32.5 \%)$, 
gastrointestinal problems $(29.2 \%)$, immune system problems (31.4\%), and other $(2.0 \%)$.

Thus, across the general population and each of the vulnerable sub-populations, the most frequently reported adverse health effects are respiratory problems, mucosal symptoms, migraine headaches, skin problems, and asthma attacks.

For each type of health problem, the frequencies are higher for vulnerable sub-populations. For instance, respiratory problems are reported by $37.7 \%$ of asthmatic individuals and $44.7 \%$ of autistic individuals when exposed to fragranced products, compared with $16.7 \%$ of the general population (Steinemann and Goodman 2019; Steinemann et al. 2018; Steinemann 2018a, b). Also, migraine headaches are reported by $22.6 \%$ of asthmatic individuals and $42.9 \%$ of autistic individuals when exposed to fragranced products, compared with $12.6 \%$ of the general population (Steinemann and Nematollahi 2020; Steinemann 2018a, b).

\section{Disabling health effects}

Health effects from exposure to fragranced products can be so severe as to be considered disabling, according to legislative criteria for disability in each country. Across the four countries, $9.5 \%$ of the general population $(17.2 \%, 5.6 \%, 7.1 \%$, $8.0 \%$ ), representing $29.1 \%$ of fragrance sensitive individuals $(49.5 \%, 17.1 \%, 25.5 \%, 24.2 \%)$, report health effects that could be considered disabling, according to the disability legislation in each country (ADAAA 2008; DDA 1992; EA 2010; DA 2008).

For vulnerable individuals, percentages of disabling effects are higher. For $24.1 \%$ of asthmatic individuals $(40.3 \%$, $15.0 \%, 20.1 \%, 20.8 \%$ ) and $72.8 \%$ of autistic individuals $(85.4 \%, 82.4 \%, 54.5 \%, 69.0 \%)$, health problems from fragranced products can be potentially disabling.

\section{Loss of workdays and loss of jobs}

Among the general population across the four countries, 9.0\% of adults have lost workdays or lost a job, in the previous year, due to illness from fragranced product exposure in the workplace $(15.1 \%, 7.7 \%, 6.3 \%, 6.7 \%)$. For vulnerable individuals, the percentages are higher: $20.6 \%$ of asthmatic individuals and $59.4 \%$ of autistic individuals have lost workdays or lost a job, in the past year, due to fragranced product exposure in the workplace. (Steinemann 2019b.)

Personal costs due to these lost workdays and lost jobs, in the past year, ranged from an estimated $\$ 86$ billion to $\$ 206$ billion, with a midrange value of $\$ 146$ billion (in terms of 2016 USD). Losses estimated for each country (US, AU, UK, SE) are as follows (midrange value, 2016 USD): $\$ 132$ billion, $\$ 2.7$ billion, $\$ 10.5$ billion, $\$ 900$ million. (Steinemann 2019b.)

Given the population of 33.9 million people who have lost workdays or a job due to fragranced product exposure (30.2 million, 1.1 million, 2.2 million, 0.4 million), this represents an average annual cost of $\$ 4300$ USD per person. Costs are estimated for direct personal expenses only and do not include other costs to individuals, employers, the health care system, and the broader society, associated with loss of productivity and loss of employment. (Steinemann 2019a, b.)

\section{Loss of societal access}

Fragranced product exposures, or the potential for exposures, are associated with loss of societal access:

Among the general population across the countries: $13.3 \%$ of individuals $(17.5 \%, 11.6 \%, 12.1 \%, 12.0 \%)$ are unable or reluctant to use the restrooms in a public place, because of the presence of an air freshener, deodorizer, or scented product; $10.4 \%$ of individuals $(14.1 \%, 10.3 \%, 10.3 \%, 6.7 \%)$ are unable or reluctant to wash their hands with soap in a public place, because the soap is fragranced; $17.0 \%$ of individuals $(20.1 \%$, $16.7 \%, 13.1 \%, 8.1 \%$ ) report that if they enter a business, and smell air fresheners or some fragranced product, they want to leave as quickly as possible; and $16.0 \%$ of individuals $(22.7 \%$, $15.0 \%, 13.5 \%, 12.6 \%$ ) have been prevented from going to some place because they would be exposed to a fragranced product that would make them sick.

For vulnerable sub-populations: $26.4 \%$ of asthmatic individuals and $62.1 \%$ of autistic individuals are unable or reluctant to use the restrooms in a public place, because of the presence of an air freshener, deodorizer, or scented product; $21.9 \%$ of asthmatic individuals and $59.8 \%$ of autistic individuals are unable or reluctant to wash their hands with soap in a public place, because the soap is fragranced; $31.6 \%$ of asthmatic individuals and $58.7 \%$ of autistic individuals report that if they enter a business, and smell air fresheners or some fragranced product, they want to leave as quickly as possible; and $32.9 \%$ of asthmatic individuals and $66.7 \%$ of autistic individuals have been prevented from going to some place because they would be exposed to a fragranced product that would make them sick.

Across all types of exposures, the percentages of individuals adversely affected are higher for vulnerable sub-populations. Practical implications of the results are compelling. For instance, more than one-fourth of asthmatic individuals and one-half of autistic individuals are prevented from using public restrooms that have air fresheners. Although washing hands with soap is intended to reduce health risks, individuals may be prevented from washing hands with soap due to health risks associated with fragrance in the soap. Further, a store 
using an air freshener or fragranced product may actually turn away rather than attract customers.

\section{Product emissions as air pollutants}

Fragranced consumer products can be a primary source of indoor air pollutants (Steinemann 2017b). In studies of indoor environments around the world, fragranced product chemicals (such as limonene) are consistently among the most prevalent and highest concentrations among pollutants (e.g., Goodman et al. 2017; Jia et al. 2008; Wang et al. 2017). In addition to being a primary source of indoor pollutants, fragranced products have been implicated as major contributors to outdoor air pollution (e.g., McDonald et al. 2018). Thus, in an interesting development, fragranced consumer products used indoors have received regulatory attention because of the ability of product emissions to migrate outdoors and affect ambient air quality (CARB 2019).

\section{Product ingredients and disclosure}

Ingredients in fragranced consumer products are not required to be specifically and fully disclosed, in any country (Lunny et al. 2017; Steinemann 2015, 2009; Steinemann et al. 2011). Main components of the regulations are as follows:

No law in any country requires the full disclosure of all ingredients in a chemical mixture termed "fragrance," not on the product label, safety data sheet, or elsewhere. Instead, a product may list the general term "fragrance" (or another legally approved term, such as "perfume" or "parfum") instead of listing all individual ingredients in that fragrance.

No law requires that consumer products (i.e., products other than foods, drugs, and cosmetics) disclose all ingredients on the label, safety data sheet, or elsewhere. Further, these products are not even required to list the general term "fragrance."

For the other classes of consumer products (i.e., foods, drugs, and cosmetics), while they do need to list all ingredients on the label (although not on the safety data sheet), the general term "fragrance" may be used instead of the specific ingredients.

Thus, and paradoxically, a primary source of exposure to pollutants, fragranced consumer products, is exempt from full disclosure of the ingredients that contribute to the pollutants. Consequently, the public, professionals, and agencies lack information to understand the links between emissions, exposures, and effects on air quality and health.
Volatile emissions from fragranced consumer products

Notwithstanding the regulatory protections on full ingredient disclosure, products can be chemically analyzed to determine their constituents. A set of studies analyzed volatile organic compounds (VOCs) emitted from common consumer products, both fragranced and fragrance-free versions, and green and regular versions (Steinemann 2015; Nematollahi et al. 2018a, b, 2019; Steinemann et al. 2020). Products were randomly selected from stores in the US and AU, although the same or similar products are also available internationally. Product definitions for these studies are as follows:

"Fragranced products" are considered products that contain "fragrance," "parfum," "perfume," "essential oils," or an aromatic scent (even if undisclosed). "Fragrance-free" products are considered products with the claim of "fragrance-free" or "no fragrance." (To note, "unscented" products are not included in this category, because they may actually contain a fragrance to cover the scent.)

"Green products" are considered products with the claim of "green" or a related term such as "organic," "natural," "no petrochemicals," "certified green," "certified organic," or "essential oils" for the entire product or specific ingredients. "Regular products" are the products other than those in the "green" category.

The studies used gas chromatography/mass spectrometry (GC/MS) headspace analysis to identify the VOCs emitted directly from each product. The top 20 peaks (highest concentration compounds) for each product were identified from the sample chromatogram using mass spectral library matches. Further analytic details are provided in Steinemann et al. (2011) and Nematollahi et al. (2018a).

"VOC occurrences" refers to the collective number of individual VOCs or ingredients emitted from the products. "VOC identities" refers to the number of distinctly named VOCs emitted from one or more of the products.

Main results of five studies (Steinemann 2015; Nematollahi et al. 2019, 2018a, b; Steinemann et al. 2020) are provided respectively below.

(1) Study of 37 common consumer products: air fresheners, laundry products, cleaning supplies, and personal care products. The GC/MS analyses found 559 VOC occurrences, representing $156 \mathrm{VOC}$ identities. Among these VOCs, 230 VOC occurrences, representing 42 VOC identities, are classified as potentially hazardous. All products emitted potentially hazardous VOCs.

The most common fragranced product VOCs $(>80 \%$ of products) were limonene and beta-pinene. The most common fragrance-free product VOC (100\% of products) was ethanol, which was also in fragranced products. The most common 
potentially hazardous VOCs ( $>75 \%$ of products) were ethanol and limonene. No significant difference was found in the emissions of hazardous air pollutants between green fragranced products and regular fragranced products.

Fewer than $3 \%$ of the VOCs, and fewer than $6 \%$ of potentially hazardous VOCs, were disclosed on the product labels, safety data sheets, or elsewhere. Further, among the fragranced consumer products (other than cosmetics), 91\% did not disclose the presence of a "fragrance" on the label or safety data sheet. Among the fragranced cosmetics, $89 \%$ did not disclose "fragrance" on the safety data sheet, but all disclosed "fragrance" on the label, as required.

(2) Study of 134 common consumer products: air fresheners, laundry products, cleaning supplies, personal care products, and sunscreens. The GC/MS analyses found 1538 VOC occurrences, representing 338 VOC identities. Among these VOCs, 517 VOC occurrences, representing 69 VOC identities, are classified as potentially hazardous. Nearly all products (99\%) emitted potentially hazardous VOCs.

The most common fragranced product VOC (77\% of products) was limonene. The most common fragrance-free product VOC (40\% of products) was ethanol. The most common potentially hazardous VOCs ( $>40 \%$ of products) were limonene and ethanol. No significant difference was found in emissions of the most prevalent potentially hazardous VOCs between green fragranced products and regular fragranced products.

Fewer than $10 \%$ of VOCs, and fewer than $4 \%$ of potentially hazardous VOCs, were disclosed on the product labels, safety data sheets, or elsewhere.

(3) Study of 42 fragranced baby products: shampoos, lotions, hair sprays, and fragrance. The GC/MS analyses found $684 \mathrm{VOC}$ occurrences, representing $228 \mathrm{VOC}$ identities. Among these VOCs, 207 VOC occurrences, representing 43 VOC identities, are classified as potentially hazardous.

The most common fragranced baby product VOC (67\% of products) was limonene. The most common potentially hazardous VOCs ( $>55 \%$ of products) were limonene, acetaldehyde, and ethanol. No significant difference was found in the emissions of the most prevalent potentially hazardous VOCs between green fragranced baby products and regular fragranced baby products.

Fewer than $5 \%$ of VOCs, and fewer than $13 \%$ of potentially hazardous VOCs, were disclosed on the product labels, safety data sheets, or elsewhere.

(4) Study of 24 commercial essential oils. The GC/MS analyses found 589 VOC occurrences, representing 188
VOC identities. Among these VOCs, 124 VOC occurrences, representing $33 \mathrm{VOC}$ identities, are classified as potentially hazardous.

The most common essential oil VOCs ( $>70 \%$ of oils) were alpha-pinene, limonene, and acetone. The most common potentially hazardous VOCs ( $>70 \%$ of oils) were limonene and acetone. No significant difference was found in the emissions of the most prevalent potentially hazardous VOCs between natural and regular essential oils. No ingredients were disclosed on any of the essential oil labels.

(5) Study of 12 car air fresheners. The GC/MS analyses found 546 VOC occurrences, representing 275 VOC identities. Among these VOCs, 30 VOC occurrences, representing 9 VOC identities, are classified as potentially hazardous.

The most common car air freshener VOCs (> 70\% of products) were limonene, benzyl acetate, acetone, and ethanol. The most common potentially hazardous VOCs (67\% of products) were acetaldehyde and methanol. No significant difference was found in the emissions of the most prevalent potentially hazardous VOCs between green and regular car air fresheners. Fewer than $2 \%$ of VOCs, and none of the potentially hazardous VOCs, were disclosed on the product labels, safety data sheets, or elsewhere.

Most prevalent VOCs Across the five studies, the 249 products emitted collectively 3916 VOCs. The most prevalent compounds in fragranced products were terpenes (limonene, alpha-pinene, beta-pinene), which were not found in fragrancefree products. The most prevalent compound in fragrance-free products was ethanol, which was also a common compound in fragranced products.

Potentially hazardous VOCs Across the studies, the 249 products emitted collectively 1108 potentially hazardous VOCs, which represents $28 \%$ of all VOC occurrences. Nearly all products $(99 \%)$ emitted potentially hazardous VOCs. The most prevalent potentially hazardous VOCs were limonene (67\% of products), ethanol $(53 \%)$, and acetaldehyde $(44 \%)$.

Comparing VOCs emitted and ingredients listed Across the studies, on average, fewer than $4 \%$ of all VOCs, and fewer than $5 \%$ of potentially hazardous VOCs, were disclosed on any product label, safety data sheet, or elsewhere.

Comparing green fragranced products and regular fragranced products Also, across the studies, no significant difference was found in the emissions of the most prevalent potentially hazardous VOCs between green (organic, 
natural) fragranced products and regular fragranced products.

Comparing fragranced and fragrance-free products Across all studies, comparing emissions from fragranced and fragrancefree versions of the same types of products, the main chemical difference is the presence of terpenes (such as limonene, betapinene, and alpha-pinene) in all fragranced products, and the absence of terpenes in all fragrance-free products. This leads to a focus on terpenes.

\section{Terpenes and reaction products}

Terpenes were the most commonly and consistently emitted VOCs from fragranced products. As noted above, terpenes were present in all fragranced products tested, but absent in all fragrance-free products tested.

Terpenes characteristic of fragranced consumer products are among the most abundant pollutant indoors and they contribute to pollutants outdoors (Steinemann et al. 2013; Steinemann 2015). In addition to being primary emissions, terpenes react with other chemicals to generate a range of secondary and potentially hazardous pollutants. For instance, terpenes react with ozone indoors to generate pollutants such as formaldehyde and ultrafine particles (Nazaroff and Weschler 2004). Terpenes react with nitrogen oxides outdoors to generate ozone and secondary organic aerosols (McDonald et al. 2018).

Common terpenes in the fragranced consumer products, such as limonene, are chiral molecules: they can exist as a right-hand enantiomer (e.g., d-limonene), a left-hand enantiomer (e.g., l-limonene), or a mixture. Chiral molecules found in nature are usually and predominantly one enantiomer or another, whereas chiral molecules that are synthetized are usually a mixture of enantiomers. For a specific chiral molecule, individual enantiomers and their mixtures can have the same chemical structure but different biological effects. An interesting area for scientific exploration is the potential difference in effects of different enantiomeric forms and sources of chiral fragrance molecules.

The paper now turns to promising approaches to reduce emissions, exposures, and effects.

\section{Fragrance-free products}

Fragrance-free products can offer similar functionality but without the potential issues associated with fragranced products. For instance, a cleaning or disinfection product may be similarly effective at its primary function without the added fragrance. Further, changes from fragranced to fragrance-free products can reduce terpenes emissions in a relatively short time period. For instance, changing from fragranced to fragrance-free laundry products can reduce concentrations of fragrance chemicals (i.e., limonene) emitted from dryer vents by up to $99 \%$ within 4 weeks (Goodman et al. 2019b). While the term "fragrance-free products" is not intended to imply emissions-free products, they do offer an option for consumer product functionality without the fragrance compounds.

In addition, removing or discontinuing use of fragrance products can also reduce emissions and exposures. For instance, removing or turning off air fresheners in a restroom can reduce concentrations of fragrance chemicals within indoor environments by up to $96 \%$ within 2 weeks (Goodman et al. 2019a). Because fragrance molecules can adhere to surfaces during product use and be re-emitted later, even without the product in use, reduction may not be $100 \%$ immediately, but fragrance compound concentrations can decrease with time.

Moreover, the fact that fragranced products can constitute a barrier to participation in society can come under the auspices of disability legislation. The change to a fragrance-free product, the removal of the fragranced product, or a modification in facilities or operations to mitigate fragrance exposure, for instance, could be considered forms of reasonable accommodation. To this end, fragrance-free practices and policies have been implemented across the countries to accommodate sensitive and vulnerable individuals, as well as to reduce potential health risks and create a more healthful indoor air environment for all (Steinemann 2019a).

\section{Preferences for fragrance-free policies and fragrance-free environments}

Nationally representative population surveys, across the four countries (US, AU, UK, SE), found that more people, at least twice as many, prefer fragrance-free environments to fragranced environments, such as workplaces, health care facilities and professionals, hotels, and airplanes. Among vulnerable sub-populations, preferences for fragrance-free environments are even higher. Interestingly, even among individuals who do not report fragrance sensitivity, a majority of these non-fragrance sensitive individuals would nonetheless prefer fragrance-free environments. Specific results are as follows (Steinemann 2018a, b, 2019a, b.)

For workplaces: $47.8 \%(53.1 \%, 42.8 \%, 44.7 \%, 50.7 \%)$ of the general population would support a fragrance-free policy in the workplace, compared with $20.4 \%(19.7 \%, 22.2 \%$, $23.3 \%, 6.4 \%$ ) that would not. Also, $56.7 \%$ of asthmatic individuals would support fragrance-free workplace policies, compared with $17.7 \%$ that would not; $65.5 \%$ of autistic individuals would support fragrance-free workplace policies, compared with $24.0 \%$ that would not; and $40.4 \%$ of nonfragrance sensitive individuals would support fragrance-free 
workplace policies, compared with $23.4 \%$ that would not. Thus, more than twice as many individuals would support (than would not) fragrance-free policies in workplaces.

For health care: $51.4 \%(54.8 \%, 43.2 \%, 43.3 \%, 64.1 \%)$ of the general population would prefer that health care facilities and health care professionals be fragrance-free, compared with $22.1 \%(22.4 \%, 25.2 \%, 26.7 \%, 14.0 \%)$ that would not. Also, $62.3 \%$ of asthmatic individuals would prefer fragrancefree health care, compared with $18.3 \%$ that would not; $77.2 \%$ of autistic individuals would prefer fragrance-free health care, compared with $16.4 \%$ that would not; and $42.3 \%$ of nonfragrance sensitive individuals would prefer fragrance-free health care, compared with $26.3 \%$ that would not. Thus, more than twice as many individuals would support (than would not) fragrance-free health care facilities and fragrance-free health care professionals.

For hotels: $60.7 \%(55.6 \%, 55.6 \%, 53.8 \%, 77.7 \%)$ of the general population would prefer hotels without fragranced air, compared with $22.1 \%(27.8 \%, 22.7 \%, 28.1 \%, 9.8 \%)$ with fragranced air. Also, $65.8 \%$ of asthmatic individuals would prefer hotels without fragranced air, compared with $22.7 \%$ with fragranced air; $52.1 \%$ of autistic individuals would prefer hotels without fragranced air, compared with $38.1 \%$ with fragranced air; and $53.7 \%$ of non-fragrance sensitive individuals would prefer hotels without fragranced air, compared with $25.1 \%$ with fragranced air. Thus, more than twice as many individuals would prefer hotels without fragranced air than with fragranced air.

For airplanes: $64.8 \%$ of the general population would prefer airplanes without fragranced air, compared with $16.1 \%$ (59.2\%, $57.7 \%, 61.9 \%, 80.2 \%$ ) with fragranced air. Also, $68.8 \%$ of asthmatic individuals would prefer airplanes without fragranced air, compared with $17.3 \%$ with fragranced air; $48.4 \%$ of autistic individuals would prefer airplanes without fragranced air, compared with $41.2 \%$ with fragranced air; and $59.5 \%$ of nonfragrance sensitive individuals would prefer airplanes without fragranced air, compared with $17.3 \%$ with fragranced air. Thus, more than twice as many individuals would prefer airplanes without fragranced air than with fragranced air.

In summary, across all settings (workplaces, health care facilities and health care professionals, hotels, and airplanes), more than twice as people prefer fragrance-free to fragranced environments. Even a majority of individuals who do not report fragrance sensitivity would nonetheless prefer fragrancefree environments. These findings are juxtaposed with trends of putting fragranced air through indoor environments, even at potential risks to individuals who can experience severe health effects from exposure.

\section{Future research}

This fragrance phenomenon is a puzzle, and this article sought to investigate the pieces and bring them together to provide new insights and directions. This domain also provides a rich area for research. Future questions for exploration include the following:

- What chemicals or mixtures of chemicals could be associated with the reported adverse effects?

- What are possible differences in effects between different forms (enantiomeric compositions) and different sources (natural, synthesized) of chiral terpenes as found in products?

- How can specific sources of fragrance chemicals that affect indoor and outdoor air quality and health, such as fragranced laundry products emitted from dryer vents, be mitigated or regulated?

- What policies or laws can help protect individuals affected by fragranced products from involuntary exposures, health risks, and loss of societal access due to secondhand scents?

- What are relative benefits for air quality and health of implementing fragrance-free policies?

- Given that fragrance formulations are extensively tested for safety, yet fragrance is associated with reports of adverse health effects, how and why could this occur?

Acknowledgments I deeply thank John Barrie, Nigel Goodman, and Neda Nematollahi for their exceptionally thoughtful and helpful reviews of the paper. I also thank Dynata (Survey Sampling International) for their superb work on the surveys. I greatly appreciate the anonymous reviewers of this paper. Finally, this article is written as a tribute and with gratitude to the Founder, Emeritus Editor-in-Chief, Professor Yong S. Chung, who selflessly devoted his life to this journal, and helped to improve air quality, atmosphere, and health around the world.

Open Access This article is licensed under a Creative Commons Attribution 4.0 International License, which permits use, sharing, adaptation, distribution and reproduction in any medium or format, as long as you give appropriate credit to the original author(s) and the source, provide a link to the Creative Commons licence, and indicate if changes were made. The images or other third party material in this article are included in the article's Creative Commons licence, unless indicated otherwise in a credit line to the material. If material is not included in the article's Creative Commons licence and your intended use is not permitted by statutory regulation or exceeds the permitted use, you will need to obtain permission directly from the copyright holder. To view a copy of this licence, visit http://creativecommons.org/licenses/by/4.0/.

\section{References}

Americans with Disabilities Act Amendments Act of 2008 (ADAAA) (2008) 42 U.S. Code $\S 12102$ - Definition of disability (Pub. L. 101-336, § 3, July 26, 1990, 104 Stat. 329; Pub. L. 110-325, $\S 4(a)$, Sept. 25, 2008, 122 Stat. 3555.) Available at: https://www. law.cornell.edu/uscode/text/42/12102. Accessed September 5, 2020

CARB (2019) California Air Resource Board, The regulation for reducing emissions from consumer products. Available at: https:// ww2.arb.ca.gov/our-work/programs/consumer-products-program/ current-regulations. Accessed September 5, 2020 
Discrimination Act (Diskrimineringslagen) (DA) (2008) SFS No: 2008: 567, Section 5:4 Definition of disability https://www.do.se/lag-ochratt/diskrimineringslagen/ Accessed September 5, 2020

Disability Discrimination Act (DDA) (1992) Australian government. Act no. 135 of 1992 , https://www.legislation.gov.au/Series/ C2004A04426. Accessed September 5, 2020

Equality Act (EA) (2010) Chapter 15. Parliament of the United Kingdom. https://www.legislation.gov.uk/ukpga/2010/15/pdfs/ukpga 20100015_en.pdf. Accessed September 5, 2020

Goodman NB, Steinemann A, Wheeler AJ, Paevere PJ, Cheng M, Brown SK (2017) Volatile organic compounds within indoor environments in Australia. Build Environ 122:116-125

Goodman N, Nematollahi N, Agosti G, Steinemann A (2019a) Evaluating air quality with and without air fresheners. Air Qual Atmos Health 13(1):1-4

Goodman NB, Wheeler AJ, Paevere PJ, Agosti G, Nematollahi N, Steinemann A (2019b) Emissions from dryer vents during use of fragranced and fragrance-free laundry products. Air Qual Atmos Health 12(3):289-295

IFRA (International Fragrance Association) (2020a) About the IFRA standards. https://ifrafragrance.org/safe-use/introduction-enjoyconfidence. Accessed 27 August 2020

IFRA (International Fragrance Association) (2020b) IFRA transparency list. https://ifrafragrance.org/initiatives/transparency/ifratransparency-list. Accessed 27 August 2020

Jia C, Batterman S, Godwin C (2008) VOCs in industrial, urban and suburban neighborhoods, part 1: indoor and outdoor concentrations, variation, and risk drivers. Atmos Environ 42(9):2083-2100

Lunny S, Nelson R, Steinemann A (2017) Something in the air but not on the label: a call for increased regulatory ingredient disclosure for fragranced consumer products. Univ New South Wales Law J 40(4):1366-1391

McDonald BC, De Gouw JA, Gilman JB, Jathar SH, Akherati A, Cappa CD, Jimenez JL, Lee-Taylor J, Hayes PL, McKeen SA, Cui YY (2018) Volatile chemical products emerging as largest petrochemical source of urban organic emissions. Science 359(6377):760-764

Nazaroff WW, Weschler CJ (2004) Cleaning products and air fresheners: exposure to primary and secondary air pollutants. Atmos Environ 38(18):2841-2865

Nematollahi N, Doronila A, Mornane P, Duan A, Kolev SD, Steinemann A (2018a) Volatile chemical emissions from fragranced baby products. Air Qual Atmos Health 11(7):785-790

Nematollahi N, Kolev SD, Steinemann A (2018b) Volatile chemical emissions from essential oils. Air Qual Atmos Health 11(8):949954

Nematollahi N, Kolev S, Steinemann A (2019) Volatile chemical emissions from 134 common consumer products. Air Qual Atmos Health 12(11):1259-1265

Steinemann AC (2009) Fragranced consumer products and undisclosed ingredients. Environ Impact Assess Rev 29(1):32-38

Steinemann A (2015) Volatile emissions from common consumer products. Air Qual Atmos Health 8(3):273-281
Steinemann A (2016) Fragranced consumer products: exposures and effects from emissions. Air Qual Atmos Health 9(8):861-866

Steinemann A (2017a) Health and societal effects from fragranced consumer products. Prev Med Rep 5:45-47

Steinemann A (2017b) Ten questions concerning air fresheners and indoor built environments. Build Environ 111:279-284

Steinemann A (2018a) Fragranced consumer products: effects on asthmatics. Air Qual Atmos Health 11(1):3-9

Steinemann A (2018b) Fragranced consumer products: effects on autistic adults in the United States, Australia, and United Kingdom. Air Qual Atmos Health 11(10):1137-1142

Steinemann A (2018c) Fragranced consumer products: sources of emissions, exposures, and health effects in the United Kingdom. Air Qual Atmos Health 11(3):253-258

Steinemann A (2018d) Exposures and effects from fragranced consumer products in Sweden. Air Qual Atmos Health 11(5):485-491

Steinemann A (2019a) Ten questions concerning fragrance-free policies and indoor environments. Build Environ 159:1-8

Steinemann A (2019b) International prevalence of fragrance sensitivity. Air Qual Atmos Health 12(8):891-897

Steinemann A (2019c) International prevalence of chemical sensitivity, co-prevalences with asthma and autism, and effects from fragranced consumer products. Air Qual Atmos Health 12(5):519-527

Steinemann A, Goodman N (2019) Fragranced consumer products and effects on asthmatics: an international population-based study. Air Qual Atmos Health 12(6):643-649

Steinemann A, Nematollahi N (2020) Migraine headaches and fragranced consumer products: an international population-based study. Air Qual Atmos Health 7:1-4

Steinemann AC, MacGregor IC, Gordon SM, Gallagher LG, Davis AL, Ribeiro DS, Wallace LA (2011) Fragranced consumer products: chemicals emitted, ingredients unlisted. Environ Impact Assess Rev 31(3):328-333

Steinemann AC, Gallagher LG, Davis AL, MacGregor IC (2013) Chemical emissions from residential dryer vents during use of fragranced laundry products. Air Qual Atmos Health 6(1):151-156

Steinemann A, Wheeler AJ, Larcombe A (2018) Fragranced consumer products: effects on asthmatic Australians. Air Qual Atmos Health 11(4):365-371

Steinemann A, Nematollahi N, Weinberg JW, Flattery J, Goodman N, Kolev S (2020) Volatile chemical emissions from car air fresheners. Air Qual Atmos Health 4:1-6

Wang CM, Barratt B, Carslaw N, Doutsi A, Dunmore RE, Ward MW, Lewis AC (2017) Unexpectedly high concentrations of monoterpenes in a study of UK homes. Environ Sci Process Impacts 19(4): $528-537$

Publisher's note Springer Nature remains neutral with regard to jurisdictional claims in published maps and institutional affiliations. 